\title{
Analysis of Group ICA functional connectivity of task-driven fMRI: application to language processes in adults with auditory deprivation
}

Antonio Napolitano, Martina Andellini, Vittorio Cannatà, Francesco Randisi, Bruno Bernardi, Marianna Castrataro, Giovanni Pezzulo, Pasquale Rinaldi, Maria Cristina Caselli, Laura Barca

Background and purpose: An increasing number of functional neuroimaging studies are examining the brain networks functionally connected while performing a cognitive task ${ }^{1,2}$, or simply during rest ${ }^{3,4}$. Few studies investigated the effect of language task on FC (functional connectivity) thus showing that the experiment-specific variations onto the basic language-related network only add a minor part of the fluctuations seen ${ }^{5}$. Studies using functional MRI and MEG suggests early involvement of brain regions devoted to speech-motor output also in task requiring covert word recognition ${ }^{6,7}$. The aim of this study is to explore the impact of auditory deprivation and communication mode on written language processing. At a behavioral level mode of communication modulates the recognition of written language, with deaf signers (i.e., preferentially using a signed language to communicate) recognizing words more rapidly than strings of consonant ${ }^{8}$. No such effect was present for deaf using spoken language (i.e.,deaf individuals using the oral modality with less or no competence in sign language). Evidence of such difference might be expected in functional connectivity networks.

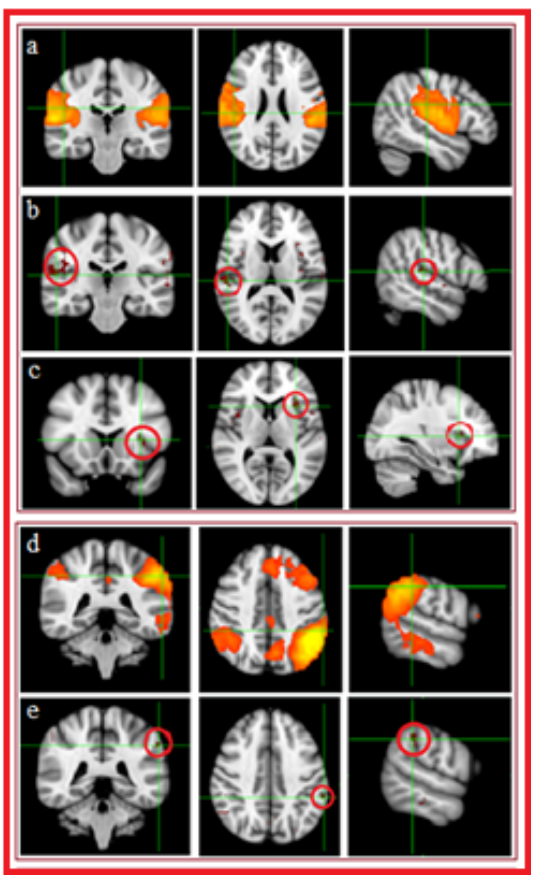

Materials and methods: All research procedures were approved by the Medical Ethics Committee of the Bambino Gesù Pediatric Hospital. Twenty adult individuals were recruited for this study. This study uses functional magnetic resonance imaging (fMRI) to examine brain activation of deaf signer subjects $(\mathrm{N}=7)$, deaf preferentially using spoken Italian $(\mathrm{N}=7)$ and a control group of hearing monolingual $(\mathrm{N}=6)$ in a visual task. All fMRI studies were performed on a 1,5 T Philips Achieva scanner. Prior to the functional run, a high-resolution T1-wheithed, structural image for each subjects was obtained by using an MPRAGE sequence with a voxel size of $1 \mathrm{~mm} 3(\mathrm{TR}=25 \mathrm{~ms}, \mathrm{TE}=$ $4,6 \mathrm{~ms}, \mathrm{FA}=30^{\circ}, \mathrm{FOV}=288 \times 288 \mathrm{~mm} 2$, matrix $=288 \times 288$, slice thickness $=1 \mathrm{~mm}$, axial slices $=175$ ). Then functional images were acquired with T2*- weighed EPI sequence with 360 dynamics. ained with $\mathrm{TR}=3000 \mathrm{~ms}, \mathrm{TE}=50 \mathrm{~ms}, \mathrm{FA}=90^{\circ}, \mathrm{FOV}=256 \times 256 \mathrm{~mm} 2$, matrix $64 \times 64$. The experimental procedure during fMRI data acquisition provided visual stimuli whereby words and unpronounceable strings of consonants were alternately presented in equal measure among all participants. They were asked to attend the stimuli and to read the words.

Functional connectivity analysis: Structural data Intensity normalization and brain extraction were carried out using Freesurfer (http://surfer.nmr.mgh.harvard.edu/). The resulting skull-stripped images were aligned to MNI 152 standard space using nonlinear registration as implemented in ANTs (http://stnava.github.io/ANTs/). Functional images were brain-extracted and motion-corrected using FSL (www.fmrib.ox.ac.uk/fsl).. The fMRI resulting volumes were registered to individual's structural scan and registered to Montreal Neurological Institute-152 standard space using ANTs nonlinear coregistration. Temporal concatenation Group ICA was carried out using probabilistic ICA as implemented in FSL's MELODIC package to identify global, distinct independent patterns of functional connectivity in the entire subject population limiting the number of independent components (ICs) to 100. The analysis for the difference between groups was carried out using an FSL dual regression technique. The statistical analysis was performed by using FSL's randomize for nonparametric permutation testing, with 5000 permutations, Threshold Free Cluster Enhanced (TFCE) technique and with a control for multiple comparison $(\mathrm{p}<0.05)^{9}$.

Results: The results show increase activity in intrinsic connectivity networks among deaf signers compared to deaf using spoken language in two networks typically related to cognitive and language tasks ${ }^{10,11}$. As shown in Figure 1 , increased connectivity was found in: a,b,c) auditory network (comprising primary and association auditory cortices, associated to action-execution-speech, cognition-language-speech, and perception-audition paradigms ); d, e) fronto-parietal network (corresponding to cognition-language paradigms).

Discussion. This is the first functional connectivity study exploring differences in brain networks involved in recognition of written language by deaf adults using different communication mode (i.e., a manual/signed language and an oral/spoken language). Overall, the functional networks extracted are similar to functional networks related to language and cognitive task, extracted from BrainMap and resting fMRI study ${ }^{10,11}$. Although preliminary, results point to a key role of communication mode (gestural vs spoken) in shaping the connectivity of networks involved in processing written language, strengthening the relation between action on perception. Furthermore, the results might corroborate the idea of the existence of a very basic network that acts as a general framework for language processing.

Reference:(1) Calhoun, V.D et al. Hum Brain Mapp, 2008. 29(7): p. 828-38. (2) Schmithorst, V.J. et al. Neuroimage, 2004. 22(3): p. 1414-20. (3) Damoiseaux, J.S., et al. Proc Natl Acad Sci U S A, 2006. 103(37): p. 13848-53. (4) Mantini, D., et al. Proc Natl Acad Sci U S A, 2007. 104(32): p. 13170-5. (5) Lohmann, G., et al. Cereb Cortex, 2010. 20(6): p. 1286-92. (6) Pammer, K. et al., Neuroimage, 2004. 22: p. 1819-1825. (7) Barca, L. et al., Brain and Language, 2011. 118: p. 53-71. (8) Barca, L. et al., PLoS ONE, 2013. 8(3): e59080. (9) Smith, S.M. and T.E. Nichols. Neuroimage, 2009. 44(1): p. 83-98. (10) Laird, A.R., et al., J Cogn Neurosci, 2011. 23(12): p. 4022-37. (11) Smith, S.M., et al., Proc Natl Acad Sci U S A, 2009. 106(31): p. $13040-5$. 